\title{
Erratum to: Efficacy of erlotinib in patients with relapsed gliobastoma multiforme who expressed EGFRVIII and PTEN determined by immunohistochemistry
}

\author{
Oscar Gallego - M. Cuatrecasas - M. Benavides • P. P. Segura • \\ A. Berrocal - N. Erill $\cdot$ A. Colomer $\cdot$ M. J. Quintana $\cdot$ \\ C. Balaña $\cdot$ M. Gil $\cdot$ A. Gallardo $\cdot$ P. Murata $\cdot$ A. Barnadas
}

Published online: 28 October 2014

(C) Springer Science+Business Media New York 2014

\section{Erratum to: J Neurooncol (2014) 116:413-419 \\ DOI 10.1007/s11060-013-1316-y}

The last author's affiliation was incorrect in the original publication. Dr. Barnadas made his contribution to the study as a professor at the Universitat Autónoma de Barcelona.
The online version of the original article can be found under doi:10.1007/s11060-013-1316-y.

O. Gallego $(\bowtie) \cdot$ M. J. Quintana · P. Murata Medical Oncology Service, Santa Creu i Sant Pau Hospital, Sant Antoni $\mathrm{M}^{\circ}$ Claret $\mathrm{n}^{\circ}$ 167, 08025 Barcelona, Spain

e-mail: secretaria@geino.es; ogallego@santpau.cat

M. Cuatrecasas

Pathology Department, Hospital Clinic, Barcelona, Spain

M. Benavides

Medical Oncology Service, Carlos Haya Hospital, Málaga, Spain

P. P. Segura

Medical Oncology Service, San Carlos University Hospital, Madrid, Spain

A. Berrocal

Medical Oncology Service, Valencia General University

Hospital Consortium, Valencia, Spain

N. Erill · A. Colomer

Althia Health, Barcelona, Spain

\author{
C. Balaña \\ Medical Oncology Service, Catalonian Institute of Oncology \\ (ICO), Germans Trias i Pujol Hospital, Badalona, Barcelona, \\ Spain \\ M. Gil \\ GEINO (Spanish Neuro-Oncology Research Group, Grupo \\ Español de Investigación en Neuro-oncologia), Medical \\ Oncology Service, Catalonian Institute of Oncology (ICO), \\ L'Hospitalet de Llobregat, Barcelona, Spain
}

\section{A. Gallardo}

Pathology Department, Clinica Girona, Girona, Spain

A. Barnadas

Universitat Autónoma de Barcelona, Barcelona, Spain 